\title{
Communication
}

\section{Carbon nanofibres from fructose using light-driven high-temperature spinning disc processor}

\author{
Hai-bo Lu, ${ }^{\text {a,b }}$ Ramiz A. Boulos, ${ }^{\mathrm{c}}$ Benjamin C.Y. Chan, ${ }^{\mathrm{a}, \mathrm{b}}$ Christopher T. Gibson, ${ }^{\mathrm{c}}$ Xiaolin Wang, ${ }^{\mathrm{d}}$ Colin L. \\ Raston, ${ }^{\mathrm{c}^{*}}$ and Hui Tong Chua ${ }^{\mathrm{a}^{*}}$
}

\author{
5 Received (in $X X X, X X X)$ Xth $X X X X X X X X X 20 X X$, Accepted $X$ th $X X X X X X X X X 20 X X$ \\ DOI: 10.1039/b000000x
}

Novel high flux bright light-driven high temperature spinning disc processor operating at $\sim 720{ }^{\circ} \mathrm{C}$ can effectively synthesise carbon nanofibres from fructose, a natural 10 feedstock, in polyethylene glycol-200, with the reaction time within minutes and with multiple reactor passes being a pivotal operating parameter in controlling the growth of the fibres.

15

Following the discovery of carbon nanotubes (CNT) produced by the arc discharge technique, ${ }^{1}$ substantial attention has been invested towards the synthesis, formation mechanism and properties of novel carbon nanomaterials, such as carbon nano 20 onions, ${ }^{2}$ carbon tubes, ${ }^{3}$ metal-included carbon nanocapsules, ${ }^{4}$ and films. With their unique structure, mechanical and electrical properties, nanoscale carbon materials have become an advent for new technologies with applications in gas storage, ${ }^{5}$ gas sensors, ${ }^{6}$ lithium-ion batteries, ${ }^{7}$ photo-voltaic devices, ${ }^{8}$ ${ }_{25}$ separation and filtration media, ${ }^{9}$ and many more. ${ }^{10}$

A number of methods for preparing carbon nanomaterials have been developed, including but not limited to arc discharge, ${ }^{1 \mathrm{~b}}$ laser ablation, ${ }^{11}$ chemical vapour deposition (CVD), electrolysis method ${ }^{12}$ and catalytic cracking. 2 Recently new forms of carbon 30 have been reported, including carbon nanoparticles, ribbon-like structures and films, which are generated by the carbonization of polymers, ${ }^{13}$ e.g. polyvinyl chloride, aromatic polyimide film and polyvinyl alcohol, in the presence of various catalysts. In addition, carbon nanosheets have been generated from hemp, ${ }^{14}$

35 and carbonaceous microspheres from saccharides, using hydrothermal processing. ${ }^{15}$ The first three of the above techniques, namely arc discharge, laser ablation, and CVD are some of the most common methods in research and industrial manufacturing environments.

40 However, the potential applications of carbon nanomaterials suffer from limitations associated with existing processing methods. For example, the conventional arc discharge method usually involves high-purity graphite electrodes, metal powders, and high-purity He and Ar gases. Thus the cost of production of 45 carbon nanomaterials is high. Moreover, by-products such as polyhedral graphite particles, encapsulated metal particles, and amorphous carbon are also formed. The use of laser techniques to generate carbon nanomaterials mandates the addition of metal particles as catalysts to the graphite targets which affects the
50 purity of the material. The formation of non-uniform material

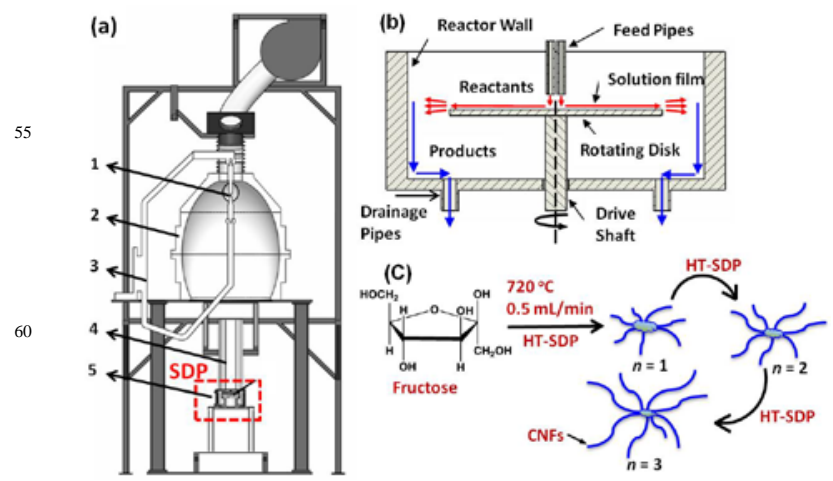

Figure 1 (a) Schematic of the light-driven HT-SDP: (1) $7 \mathrm{~kW}$ xenon short arc discharge lamp, (2) Hemi-ellipsoidal reflector, (3) Manual XYZ micromanipulator, (4) Hexagonal kaleidoscope flux homogenizer, and (5) SDP, (b) schematic of SDP in Fig 1a, and (c) the growth of CNFs via multiple $n$ passes of fructose in PEG 200 through SDP.

65 under similar conditions is also an issue. ${ }^{16}$ The CVD process is the dominant commercial technique for the fabrication of carbon nanomaterisls. ${ }^{17}$ The major benefit of CVD for the synthesis of carbon nanomaterials lies in its scalability. However, the CVD synthesis of carbon nanomaterials typically requires temperatures 70 up to $900^{\circ} \mathrm{C}$ with metal particles as the catalyst. The liquid-phase electrolytic method has not been widely used. ${ }^{18}$

A new and simple method to gain access to carbon nanomaterials is therefore warranted. To this end, we have developed a novel, rapid and facile synthetic strategy to prepare 75 carbon nanomaterials, using a high flux bright light-driven high temperature spinning disc processor (HT-SDP), Figure $1 .{ }^{19} \mathrm{We}$ have targeted fructose as a benign and cheap carbon source, with polyethylene glycol-200 (PEG 200) as a benign reaction medium, and the formation of carbon nanomaterials is void of 80 catalyst. Compared to other methods, HT-SDP exploits the high shear force within the dynamic thin liquid films (typically less than $200 \mu \mathrm{m}$ ), ${ }^{19}$ over the surface of a rotating disc to enhance reactions. Such technology reduces the duration of chemical synthesis compared to traditional batch processing, and can exert 85 control over the shape, size and morphology of the resulting nanoparticles. ${ }^{20}$ In addition, the highly directional heating was achieved by means of focusing the light from a high intensity 
xenon short arc discharge lamp onto the surface of the spinning disc via a homogeniser to ensure uniform surface heat flux. The technology can also achieve a high temperature over the surface of the disc whilst maintaining a sharp temperature gradient at the 5 reactor wall, as well as a high temperature annealing environment that favours the formation of nanomaterials. ${ }^{21}$

Furthermore, adopting more environmentally benign synthetic approaches mitigates energy consumption, reduces the generation of waste and the use of toxic substances, and provides 10 a safer working environment. This is in accordance with the mission of several international agencies like the US Environmental Protection Agency (EPA) to incorporate green chemistry principles across the lifecycle of a chemical product, including its design, manufacture, and use. ${ }^{22}$ At the same time it 15 can be more economically beneficial using HT-SDP. The exploration of energy-saving methods for the mass production of carbon nanostructures is significant. The synthesis of carbon nanostructures was explored via the direct carbonization of fructose as a sugar precursor dissolved in PEG 200, using HT${ }_{20}$ SDP operating at $\sim 720{ }^{\circ} \mathrm{C}$. The yield was estimated as approximately $6.25 \%$ through the centrifugation of the collected product.

The present method is a significant breakthrough and as a continuous flow process it could be readily scaled up. Moreover, 25 the findings dramatically reduces the length of time necessary for the synthesis of carbon nanostructures, which previously required lengthy periods of calcinations or pyrolysis following initial carbon nanostructures formation using other methods. The results also broaden the scope of the application of the SDP 30 which has been limited by the maximum temperature achievable of close to $200{ }^{\circ} \mathrm{C},{ }^{23}$ except for our recent work on generating anatase nanoparticles directly from titanium(IV) tetrakis(isopropoxide). ${ }^{19}$

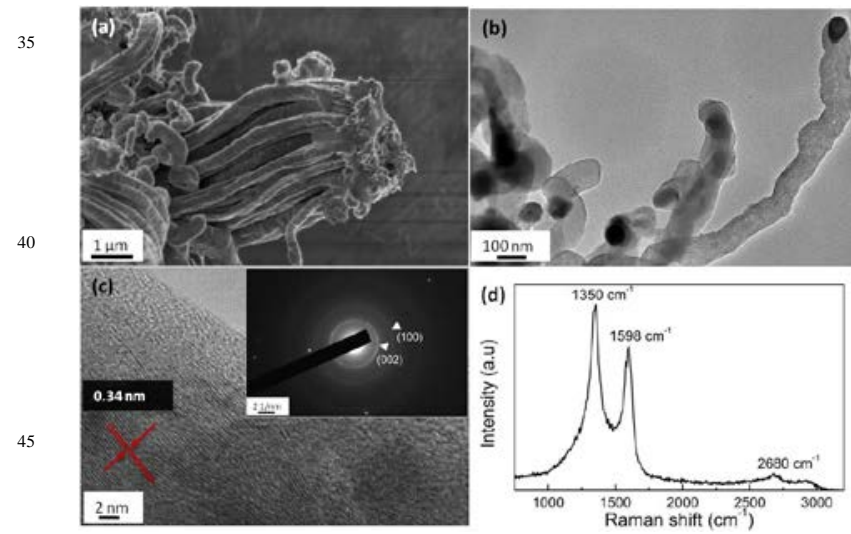

Figure 2 Microstructure of CNFs obtained from fructose in PEG-200 at $720^{\circ} \mathrm{C}$ : (a) SEM image, (b) TEM image, (c) HRTEM image and SAED pattern (inset), and (d) Raman spectra.

Fructose was dissolved in PEG 200 at a concentration of 0.01 $\mathrm{g} / \mathrm{mL}$, and directed through a jet feed close to the centre of the spinning disc, at a flow rate of $0.5 \mathrm{~mL} / \mathrm{s}, \mathrm{S} 1$, ESI†. This resulted 50 in a large quantity of entangled carbon nanofibers (CNFs) with an average diameter of $100 \mathrm{~nm}$ and a length of $\sim 4 \mu \mathrm{m}$, estimated from the SEM image, Figure 2(a). Metallic particles are attached to the caps of some of the CNFs, Figure 2(b), while others are devoid of such particles. According to the electron energy loss
55 spectroscopy (EELS) spectrum and elemental mapping, the characteristic peak of $\mathrm{Fe}$ is palpable in these metallic particles, confirming that Fe stems from the stainless steel disc, Figure S1, ESI†. The lattice fringes in HRTEM image, Figure 2(c), show the characteristics of graphitic carbon, with an inter-planar 60 spacing of about $0.34 \mathrm{~nm}$ (indicated by parallel lines) being consistent with the (002) plane lattice parameter of graphitic carbon. These lattice planes are further confirmed by the selected area electron diffraction (SAED) rings, Figure 2(c) inset. Raman spectra, Figure 2(d), had distinct peaks at 1598 and $1350 \mathrm{~cm}^{-1}$ 65 attributed to the $\mathrm{G}$ and $\mathrm{D}$ bands, respectively, of carbon based materials. The $\mathrm{G}$ band confirms the presence of graphitic carbon and the $\mathrm{D}$ band shows a high sensitivity to disorder, ${ }^{24}$ with a D/G peak intensity ratio of $\sim 1.25$ indicative of largely disordered carbon. Similar Raman spectra and D/G ratio for CNFs have 70 been observed for material generated by catalytic chemical vapour deposition of acetylene. ${ }^{25}$

The nature of the product depends on the synthetic procedure used, especially in relation to the temperature and processing time. During the process, the dehydration process is likely to be 75 at low temperature and carbonization at high temperature, affording the CNFs from fructose. ${ }^{15 b}$ Usually, the carbonization temperature of sugar is from 600 to $1600{ }^{\circ} \mathrm{C} .{ }^{26}$ When the fructose-PEG 200 solution is fed onto the SDP, it dehydrates rapidly on the spinning disc within the dynamic thin film under 80 high temperature due to the high intensity xenon short arc discharge lamp. Then carbon nuclei begin to form during the carbonization process at high temperature, and gradually grow into more ordered graphitic structures, thereby transforming fructose into CNFs, as shown schematically in Figure 1(c).

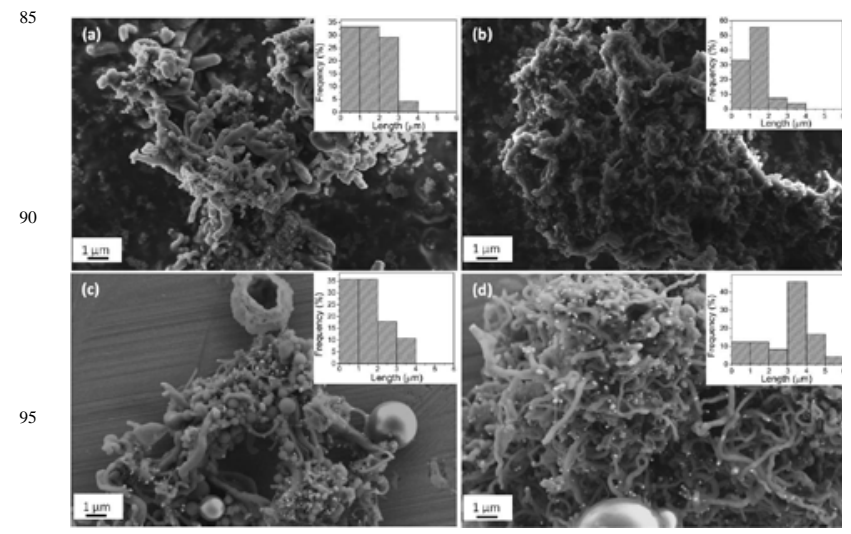

Figure 3 The morphologies and histograms (inset) of the length distribution of CNFs when (a) $n=1$, (b) $n=2$, (c) $n=3$ and (d) $n=4$.

The length of the CNFs can also be varied. This involves recycling the reaction mixture in the HT-SDP (i.e. multiple $n$ 100 passes) resulting in longer CNFs with increasing number of reactor passes, suggesting that the CNFs continue to grow as they re-flow through the HT-SDP over successive cycles, with the width of the CNFs being unchanged, Figure 3. From the histograms of the length distribution, the length increased to 4 $105 \mu \mathrm{m}$ with increased reactor passes. Our product also consists of aggregates of typical carbonaceous microspheres with diameters between $50 \mathrm{~nm}-2 \mu \mathrm{m}$, Figure 3(c-d). This morphology is also confirmed by another group, producing carbon materials by hydrothermal carbonization of cellulose. ${ }^{15}$, 27Error! Bookmark not 
defined.

In conclusion, we demonstrate a versatile method for making CNFs using a novel high bright flux light-driven HT-SDP microfluidic platform where the reaction is constrained to within 5 a dynamic thin film. The work exploits a readily available and abundant starting material, namely fructose, in the absence of a catalyst, although clearly some iron containing nanoparticles stemming from the stainless steel disc may be important in the growth of some of the CNFs. This simple and rather benign 10 approach also exerts control over the length of the CNFs. Future work will investigate the effect of different parameters of the HT-SDP on the carbon materials obtained including different temperatures and carbon precursors, e.g. different sugars or mixtures of sugars.

15 The results augment the use of the same device to fabricate small nanoparticles of anatase from a single source of titanium(IV), in a single pass at ca. $500{ }^{\circ} \mathrm{C}$, also in PEG 200, where there is no evidence of degradation of the polymer. ${ }^{19} \mathrm{An}$ important aspect of the use of a spinning disc is the short 20 residence time, typically $\leq 1 \mathrm{~s} /$ pass, which reduces the likelihood of degradation of the PEG-200. The microfluidic platform also ensures that all the precursor fructose molecules are subject to the same processing as they are transported across the disc, with the thin film ensuring uniform heating during the process, and 25 the viscous drag imparting intense shear in the dynamic thin film.

Support of this work by the Australian Research Council, The University of Western Australia, Flinders University, and The Government of South Australia, is gratefully acknowledged. The 30 microscopy analysis was carried out using facilities in the Centre for Microscopy, Characterization and Analysis, The University of Western Australia, and the Australian Microscopy and Microanalysis Research Facility, Flinders University.

\section{Notes and references}

$35{ }^{a}$ School of Mechanical and Chemical Engineering, The University of Western Australia, 35 Stirling Hwy, Crawley, WA 6009, Australia. Email: huitong.chua@uwa.edu.au

${ }^{b}$ Centre for Strategic Nano-Fabrication, School of Biomedical, Biomolecular and Chemical Sciences, The University of Western 40 Australia, WA 6009, Australia

Email: colin.raston@flinders.edu.au

${ }^{c}$ Centre for Nanoscale Science and Technology, School of Chemical and Physical Sciences, Flinders University, Bedford Park, SA 5042, Australia ${ }^{d}$ School of Engineering, The University of Tasmania, Hobart, TAS 7001, 45 Australia

† Electronic Supplementary Information (ESI) available: [details of any supplementary information available should be included here]. See DOI: $10.1039 / \mathrm{b} 000000 \mathrm{x} /$

1 (a) S. Iljima, Nature, 1991, 354, 56-58.(b) S. Iljima, and T. Ichihashi, Nature, 1993, 363, 603-605.

2 A. Cornejo, W. Zhang, L. Gao, R.R. Varsani, M. Saunders, K.S. Iyer, C.L. Raston and H.T. Chua, Chem. Eur. J., 2011, 17, 9188 - 9192.

3 X.B. Wang, W.P. Hu, Y.Q. Liu, C.F. Long, Y. Xu, S.Q. Zhou, D.B. Zhu, and L.M. Dai, Carbon, 2001, 39, 1533-1536.

4 (a) H.H Song, and X.H. Chen, Chem. Phys. Lett., 2003, 374, 400-404. (b) J.P. Huo, H.H. Song, and X.H. Chen, Carbon, 2004, 42, 31773182.

5 (a) A.C. Dillon, K.M. Jones, T.A. Bekkedahl, C.H. Kiang, D.S. Bethune, and M.J. Heben, Nature, 1997, 386, 377-379. (b) P.X. Hou, S.T. Xu, Z. Ying, Q.H. Yang, C. Liu, and H.M. Cheng, Carbon, 2003, 41, 2471-2476.
6 (a) J. Kong, N.R. Franklin, C. Zhou, M.G. Chapline, S. Peng, K. Cho, and H. Dai, Science, 2000, 287, 622-625. (b) P.G. Collins, K. Bradley, M. Ishigami, and A. Zettl, Science, 2000, 287, 1801-1804.

7 (a) S.W. Lee, N. Yabuuchi, B.M. Gallant, S. Chen, B. Kim, P.T. Hammond, and S. Yang, Nature Nanotechnol., 2010, 5, 531-537. (b) B.J. Landi, M.J. Ganter, C.D. Cress, R.A. DiLeo, and R.P. Raffaelle, Energy Environ. Sci., 2009, 2, 638-654.

8 J.R. Dahn, T. Zheng, Y. Liu, and J.S. Xue, Science, 1995, 270, 590593.

9 K.B. Jirage, J.C. Hulteen, and C.R. Martin, Science, 1997, 278, 655658.

10 (a) S.J. Trans, A.R.M. Verschueren, and C. Dekker, Nature, 1998, 393, 49-52. (b) H. Dai, E.W. Wong, and C.M. Lieber, Science, 1996, 272, 523-526. (c) M.M.J. Traecy, T.W. Ebbesen, and J.M. Gibson, Nature, 1996, 381, 678-680. (d) E.W. Wong, P.E. Sheehan, and C.M. Lieber, Science, 1997, 277, 1971-1975. (e) P. Poncharal, Z.L. Wang, D. Ugarte, and W.A. de Heer, Science, 1999, 283, 1513-1516. (f) M. Faizah, R.A. Boulos, B.Y. Hong, A. Cornejo, K. S. Iyer, L. Gao, H. T. Chua and C.L. Raston, Chem. Commun., 2012, 48, 10102-10104

11 T. Guo, P. Nikoleav, A.G. Rinzler, D. Tománek, D.T. Colbert, and R.E..Smalley, J. Phys. Chem., 1995, 99, 10694-10697.

12 (a) W.K. Hsu, J.P. Hare, M Terrones, H.W. Kroto, D.R.M. Walton, and P.J.F. Harris, Nature, 1995, 377, 687-688. (b) W.K. Hsu, J.P. Hare, M. Terrones, H.W. Kroto, and D.R.M. Walton., Chem. Phys. Lett., 1996, 262, 161-166.

13 (a) P.J.F. Harris, and S.C.Tsang, Chem. Phys. Lett., 1998, 293, 53-58. (b) M. Inagaki, K. Fujitab, Y. Takeuchia, K. Oshidac, H. Iwata, and H. Konno, Carbon, 2001, 39, 921-929. (c) Y. Kaburagi, Y. Hishiyama, H. Oka, and M. Inagaki, Carbon, 2001, 39, 593-603.

14 H. Wang, Z. Xu, A. Kohandehghan, Z. Li, K. Cui, X. Tan, T. J. Stephenson, C. K. King'ondu, C. M. B. Holt, B. C. Olsen, J. K. Tak, D. Harfield, A. O. Anyia, D. Mitlin, ACS Nano, 2013, 7, 5131-5141.

15 (a) M. Sevilla, and A.B. Fuertes, Chem. Eur. J., 2009, 15, 4195-4203. (b) Q. Wang, H. Li, L. Chen, and X. Huang, Carbon, 2001, 39, 22112214. (c) C. Yao, Y. Shin, L.Q. Wang, C. F. Windish, W. D. Samuels, B. W. Arey, C. Wang, W. M. Risen, G. J. Exarhos, J. Phys. Chem. C, 2007, 111, 15141-15145. (d) L. Zhao, N. Baccile, S. Gross, Y. Zhang, W. Wei, Y. Sun, M. Antonietti, and M. Titirici, Carbon, 2010, 48, 3778-3787.

16 D.T. Colbert, J. Zhang, S.M. McClure, P. Nikolaev, Z. Chen, J.H. Hafner, D.W. Owens, P.G. Kofula, C.B. Carfer, J.H. Weaver, A.G. Rinzler, and R.E. Smalley, Science, 1994, 266, 1218-1222.

17 C. See and A. Harris, Ind. Eng. Chem. Res., 2007, 46, 997-1012.

18 M. Terrones, Annu. Rev. Mater. Res., 2003, 33, 419-501.

19 B.C.Y. Chan, X. Wang, L.K.W. Lam, J.M. Gordon, D. Feuermann, C.L. Raston, and H.T. Chua, Chem. Engin. J., 2012, 211-212, 195199.

20 H.B. Lu, B.C.Y. Chan, X. Wang, H.T. Chua, C.L. Raston, A. AlbuYaron, M. Levy, R. Popowitz-Biro, R. Tenne, D. Feuermann, and J.M. Gordon, Nanotechnology, 2013, 24, 335603 1-7.

21 J.M. Gordon,E.A. Katz, D.Feuermann, A.A.Yaron, M.Levy, and R. Tenne, J. Mater. Chem., 2008, 18, 458-462.

22 http://www.epa.gov/greenchemistry/pubs/pgcc/presgcc.html

23 (a) Green Chemistry and Engineering (Google eBook), M. Doble, and A. Kumar, 2010, p154 (b) C. Ramshaw, and A. Aoune, Int. J. Heat and Mass Transfer, 1999, 42, 2543-2556.

24 A. Cuesta, P. Dhamelinocourt, J. Laureyns, A. Martinez-Alonso and M.D. Tascon, Carbon, 1994, 32, 1523-1532.

25 H. I. Hima, X. Xiang, L. Zhang and F. Li, J. Mater. Chem., 2008, 18, 1245-1252.

26 W. Xing, J. S. Xue, and J. R. Dahn, J Electrochem Soc., 1996, 143, 3046-3052.

27 M. Sevilla, and A.B. Fuertes, Carbon, 2009, 47, 2281-2289. 